\title{
REFLECTION CENTRALIZERS IN COXETER GROUPS
}

\author{
DANIEL ALLCOCK
}

\begin{abstract}
We refine Brink's theorem, that the non-reflection part of a reflection centralizer in a Coxeter group $W$ is a free group. We give an explicit set of generators for the centralizer, which is finitely generated when $W$ is. And we give a method for computing the Coxeter diagram for its reflection subgroup. In many cases, our method allows one to compute centralizers in one's head.
\end{abstract}

Brink proved the elegant result that the centralizer of a reflection in a Coxeter group is the semidirect product of a Coxeter group by a free group [6]. In fact this free group is the fundamental group of the component of the "odd Coxeter diagram" distinguished by the conjugacy class of the reflection. Alekseevski, Michor and Neretin [1] independently gave another approach to reflection centralizers. We will give several refinements to both papers.

The first refinement is an explicit finite set of generators for the reflection centralizer; Brink only gave explicit generators for the free part. This generating set plays a key role in the author's work [3] on Steinberg and Kac-Moody groups.

The second refinement is a method of computing the Coxeter diagram of the reflection subgroup of the centralizer. With a little effort we develop this method to the point that many centralizer computations are very easy. For example, the fact that the reflection centralizer in $W\left(E_{8}\right)$ is $W\left(E_{7}\right) \times 2$ becomes a quick mental computation. We offer many other examples, including the reflection centralizer when the Dynkin diagram is any cycle of odd edges. Our most complicated example is the reflection centralizer in Bugaenko's Coxeter group that acts cocompactly on 8-dimensional hyperbolic space [8].

Our method has some overlap with the Brink-Howlett algorithm for understanding normalizers of parabolic subgroups in Coxeter groups. (See [7, and the related 2] and [4.) However, in use it feels quite different. They present a certain groupoid, any one of whose maximal subgroups is the normalizer.

In section 1 we sketch a proof of Brink's theorem following the ideas of [1]. This proof is quite different from hers, using covering spaces and topology in place of induction on word lengths. We hope this alternate proof will be helpful to some people. In following sections we give explicit generators for the centralizer, general rules for computing the Coxeter diagram of its reflection subgroup, and many examples.

The author is grateful to the Japan Society for the Promotion of Science, the Clay Mathematics Institute and Kyoto University for their support and hospitality during this work, to R. Howlett for pointing out a mis-drawn Coxeter diagram, and to one of the referees for referring me to [1].

Date: June 27, 2013.

2000 Mathematics Subject Classification. 20F55.

Partly supported by NSF grants DMS-0600112 and DMS-1101566. 


\section{BACKGROUND AND PREVIOUS RESULTS}

We will review some standard Coxeter group theory, and some results of Brink, Howlett and Alekseevski-Michor-Neretin. Our perspective will be geometric, essentially that of Vinberg from [13. We will sketch the proofs, to unify the original approaches.

A Coxeter system means a pair $(W, S)$ where $W$ is a group and $S$ is a set of involutions generating $W$, for which the relations $\left(s s^{\prime}\right)^{o\left(s s^{\prime}\right)}=1$ suffice to present $W$, where $s, s^{\prime}$ vary over $S$ and $o\left(s s^{\prime}\right)$ means the order of $s s^{\prime}$. A relation " $\left(s s^{\prime}\right)^{\infty}=1$ " is regarded as no relation at all. As usual the Coxeter diagram of $(W, S)$, usually written $\Delta$, means the graph with vertex set $S$ and $s, s^{\prime} \in S$ joined by an edge marked $o\left(s s^{\prime}\right)$. When actually drawing diagrams we follow the standard conventions of omitting edges that would be labeled 2, omitting labels from edges that would be labeled 3 or 4 , and drawing edges that would be labeled 4 as double edges.

We use the semi-standard term "spherical" for a Coxeter system or diagram when the corresponding group is finite. This reflects the fact that the group acts naturally on a sphere, rather than say hyperbolic space. In the many places where we refer to the parity of an edge label we use the convention that $\infty$ is neither even nor odd.

Our first goal is to introduce what we call a Vinberg representation, which provides the setting for the ideas. A reflection of a real vector space means a linear transformation which pointwise fixes a hyperplane (its mirror) and negates some complementary 1-dimensional space. Now suppose $V$ is a finite-dimensional real vector space, $\Gamma$ is a subgroup of $\mathrm{GL}(V)$ generated by reflections, and $U$ is an open convex subset of $V$ which $\Gamma$ preserves and acts on properly discontinuously. One consequence of proper discontinuity is the local finiteness of the arrangement of the mirrors of all reflections in $\Gamma$. It follows that the complement of the mirrors is open. By a chamber we mean the closure in $U$ of a component of this complement. By local finiteness, the boundary of a chamber is locally polyhedral, so we may speak of its faces and their dimensions. In particular we may speak of a chamber's facets (codimension-one faces). Another consequence of proper discontinuity is that each mirror is the mirror of only one reflection of $\Gamma$, so we may speak unambiguously of the reflection across each facet of a chamber.

Theorem 1. Let $K$ be a chamber and $S$ the set of reflections across its facets. Then (i) $(\Gamma, S)$ is a Coxeter system, (ii) $\Gamma$ acts freely on the set of chambers, and (iii) every point of $U$ is $\Gamma$-equivalent to a unique point of $K$.

Proof sketch. Theorem 1 of [5, IV.4.4] addresses a slightly different situation, the geometric representation of a Coxeter group. But the proof applies verbatim to prove (i) and (ii). More specifically, consider the Coxeter system $(W, S)$ where $W$ is the abstract group with generating set $S$ and defining relations $\left(s s^{\prime}\right)^{o\left(s s^{\prime}\right)}=1$ where $o\left(s s^{\prime}\right)$ means the order of $s s^{\prime}$ as an element of $\Gamma$. Then $w\left(K^{\circ}\right) \cap K^{\circ}=\emptyset$ for all $w \in W-\{1\}$. It follows that $W \rightarrow \Gamma$ is an isomorphism, hence (i) and (ii). The proof in [5, IV.4.4] relies on its Lemma 1, which is essentially a standard-form result for the dihedral group in $\operatorname{GL}(V)$ generated by any two elements of $S$. In our case one establishes this lemma using the definition of a chamber as a component of the mirror complement, rather than the definition of the geometric representation.

For (iii) one follows the proof of Prop. 5 of [5, IV.6]. 
In this situation we call $(V, U)$ a Vinberg representation of $\Gamma$ and $(V, U, K)$ a Vinberg representation of $(\Gamma, S)$. The name honors Vinberg's proof 13 that any Coxeter system $(W, S)$ with $S$ finite admits such a representation. If $S$ is infinite then a Vinberg representation may still exist, for example by taking the reflections across the facets of a suitable infinite-sided polyhedron in hyperbolic space, or by taking a subgroup generated by reflections in a Coxeter group that admits a Vinberg representation. But it may not, for example the ascending union of the finite symmetric groups is a Coxeter group but its only finite-dimensional representation is the trivial one. One could contemplate infinite-dimensional Vinberg representations, at the cost of more care with topological concepts like proper discontinuity. In our case one can deduce results for general Coxeter systems from the corresponding results for the $|S|<\infty$ case; see corollary 6 .

For the rest of this section we will fix a Coxeter system $(W, S)$ that admits some Vinberg representation $(V, U, K)$. Our goal is to understand the $W$-centralizer of a reflection $s$, meaning an element conjugate into $S$. Although not all Coxeter systems admit Vinberg representations, most interesting ones do, and it turns out that understanding this case well enough allows us to later remove the assumption that such a representation exists. Define $W_{\Omega}$ as the subgroup of $W$ generated by the reflections in $C_{W}(s)$ other than $s$. These reflections are the same as those that preserve $s$ 's mirror $V^{s}$ and each of the two half-spaces it bounds (call one of them $\left.\frac{1}{2} V\right)$. It is easy to check that $\left(V^{s}, U^{s}\right)$ is a Vinberg representation of $W_{\Omega}$. By theorem 1, $W_{\Omega}$ is a Coxeter group. Now choose a chamber $K_{\Omega}$ for $W_{\Omega}$ in $U^{s}$ and define $\Gamma_{\Omega}$ as the subgroup of $W$ that preserves $K_{\Omega}$ and $\frac{1}{2} V$. Note that $K_{\Omega}$ has one dimension less than $K$. To avoid confusion we specify: when we speak of chambers without mentioning $W_{\Omega}$ explicitly, we always mean chambers of $W$. We have just sketched a proof of the following theorem of Howlett. (The semidirect product decomposition comes from the freeness of $W_{\Omega}$ 's action on its set of chambers.)

Theorem 2 ([12, corollaries 3 and 7], [7]). $C_{W}(s)=\langle s\rangle \times\left\langle W_{\Omega}, \Gamma_{\Omega}\right\rangle$, and the latter factor splits as the semidirect product of $W_{\Omega}$ by $\Gamma_{\Omega}$.

To understand $C_{W}(s)$ it now suffices to understand $W_{\Omega}, \Gamma_{\Omega}$ and the latter's action on the former. We begin with $\Gamma_{\Omega}$. The key to understanding it is that the interior $K_{\Omega}^{\circ}$ of $K_{\Omega}$ turns out to be the universal cover of part of the boundary of the chamber $K$ that we started with. A nice mental image of this is to fold $K_{\Omega}^{\circ}$ along its intersections with mirrors of $W$, and then wrap it around $K$ as one might wrap a Weyl-chamber-shaped gift. This idea is due to Alekseevski, Michor and Neretin [1, and we will sketch it in our language. Vinberg has informed me that he and O. Shvartsman knew of it earlier.

Given a codimension 2 face of a chamber, we say the angle there is $\pi / n$ if the product of the reflections corresponding to its two facets has order $n$. We define $X$ as the boundary of $K$ in $U$, minus those codimension 2 faces with angle $\pi /$ (even).

Proposition 3 ([1, Prop. 2.9]). The natural map $U \rightarrow U / W=K$ induces a universal covering map from $K_{\Omega}^{\circ}$ to a component of $X$, with deck group $\Gamma_{\Omega}$.

Proof sketch. The first ingredient is that $K_{\Omega}^{\circ}$ contains no codimension 3 face of any of $W$ 's chambers. (Otherwise, the face's $W$-stabilizer would be a rank 3 spherical Coxeter group, containing $s$. Every reflection in such a group centralizes some other reflection in it. The face therefore lies in the mirror of a reflection in $W_{\Omega}$, while $K_{\Omega}^{\circ}$ is disjoint from such mirrors by its definition.) Similarly, $X$ contains 
no codimension 3 face of $K$. (Such a face would correspond to a rank 3 spherical Coxeter subsystem of $S$, so some pair of the three facets involved would make angle $\pi / 2$, so the face lies in a codimension 2 face of $K$ which we discarded when defining $X$.)

The same argument shows that $K_{\Omega}^{\circ}$ contains no codimension 2 face of any of $W$ 's chambers whose angle is $\pi /($ even). And $X$ contains no codimension 2 face of $K$ with angle $\pi /$ (even) by definition.

On the other hand, if $K^{\prime}$ is a chamber of $W$ with a facet $F$ in $K_{\Omega}$, and $f$ is a facet of $F$ whose angle in $K^{\prime}$ is $\pi$ /(odd) then the interior of $f$ does lie in $K_{\Omega}^{\circ}$. This is because $f$ 's $W$-stabilizer is a dihedral group of twice odd order, and in such a group no reflection centralizes any other. Furthermore, the facet (of some other chamber) in $U^{s}$ on the other side of $f$ is equivalent under this dihedral group to the other facet of $K^{\prime}$ containing $f$ (i.e., not $F$ ). For a picture see [1, fig. 3]. This is what we referred to when comparing $K_{\Omega}^{\circ} \rightarrow K$ to wrapping a gift. Using this and the previous paragraphs one can show that $K_{\Omega}^{\circ} \rightarrow X$ is a covering map. It is a universal covering of a component of $X$ because $K_{\Omega}^{\circ}$ is convex, hence connected and simply connected.

That the deck group is $\Gamma_{\Omega}$ is the fact that if $x, y \in K_{\Omega}^{\circ}$ are $W$-equivalent, say $g(x)=y$, then they are also $\Gamma_{\Omega}$-equivalent. This can be proven by using the fact that the $W$-stabilizer of any element of $K_{\Omega}^{\circ}$ is either $\langle s\rangle$ or a dihedral group of twice odd order that contains $s$. (And that in such a group all reflections are conjugate.)

The "odd Coxeter diagram" $\Delta^{\text {odd }}$ means the graph with vertex set $S$ and $s, s^{\prime}$ joined just if $o\left(s s^{\prime}\right)$ is odd. Another way to say this is that $V^{s} \cap V^{s^{\prime}} \cap X$ is nonempty just if $s, s^{\prime}$ are joined in $\Delta^{\text {odd }}$. Using the fact that $X$ contains no codimension 3 faces of $K$, one can regard $\Delta^{\text {odd }}$ as a deformation-retract of $X$ (cf. [1, Lemma 2.8]).

It is well-known that the components of $\Delta^{\text {odd }}$ correspond to the conjugacy classes of reflections in $W$. (If elements of $S$ are oddly joined then they are conjugate in the group they generate, and if they are not joined by a chain of odd edges then they map to distinct elements of $W$ 's abelianization.) So our reflection $s \in W$ distinguishes a component of $\Delta^{\text {odd }}$, for which we write $\Delta_{s}^{\text {odd }}$, and the corresponding component of $X$. This leads to the following special case of Brink's theorem on reflection centralizers. We will recover her full result, which does not need a Vinberg representation, as corollary 7

Theorem 4 (Brink [6]). The non-reflection part $\Gamma_{\Omega}$ of the centralizer $C_{W}(s)$ is the free group $\pi_{1}\left(\Delta_{s}^{\text {odd }}\right)$.

Proof. Because $s \in S, U^{s}$ contains $s$ 's facet of $K$. By choice of the chamber $K_{\Omega}$ we may suppose it does as well. So the component $X_{s}$ of $X$ of which $K_{\Omega}^{\circ}$ is the universal cover is the one corresponding to the component of $\Delta^{\text {odd }}$ containing $s$. Proposition 3 shows that $\Gamma_{\Omega}$ is $\pi_{1}\left(X_{s}\right)$, and the homotopy-equivalence $X \simeq \Delta^{\text {odd }}$ identifies $\pi_{1}\left(X_{s}\right)$ with $\pi_{1}\left(\Delta^{\text {odd }}, s\right)$.

\section{EXPLICIT GENERATORS FOR THE CENTRALIZER}

In corollary 6 we give an explicit generating set for a reflection centralizer in any Coxeter group $W$. We need this for a forthcoming application to Steinberg and Kac-Moody groups [3], and it allows us to prove the full version of Brink's theorem 
(corollary 7). Also, if $W$ is finitely generated then so is every reflection centralizer (corollary 8).

Suppose $(W, S)$ is a Coxeter system with diagram $\Delta$ and $\gamma=\left(t_{0}, \ldots, t_{n}\right)$ is an edge-path in $\Delta^{\text {odd }}$, with $2 l_{i}+1$ being the label on the edge joining $t_{i-1}$ and $t_{i}$. Then we set

$$
p_{\gamma}:=\left(t_{1} t_{0}\right)^{l_{1}}\left(t_{2} t_{1}\right)^{l_{2}} \cdots\left(t_{n} t_{n-1}\right)^{l_{n}}
$$

(or $p_{\gamma}=1$ if $\gamma$ has length 0 ). This word is Brink's $\pi\left(t_{0}, \ldots, t_{n}\right)$. If $\gamma_{2}$ starts where $\gamma_{1}$ ends, then obviously $p_{\gamma_{1} \gamma_{2}}=p_{\gamma_{1}} p_{\gamma_{2}}$. If $u$ is a vertex of $\Delta$ evenly joined to $t_{n}$, say with edge label $2 \lambda$, then we define

$$
r_{\gamma, u}:=p_{\gamma} \cdot\left(u t_{n}\right)^{\lambda-1} u \cdot p_{\gamma}^{-1} .
$$

Whenever we refer to $r_{\gamma, u}$ we implicitly require $u$ to be evenly joined to the endpoint of $\gamma$. If $\gamma_{2}$ starts where $\gamma_{1}$ ends, then obviously $p_{\gamma_{1}} r_{\gamma_{2}, u} p_{\gamma_{1}}^{-1}=r_{\gamma_{1} \gamma_{2}, u}$.

Theorem 5. Suppose $(W, S)$ admits a Vinberg representation $(V, U, K)$ and $s \in S$.

(i) The set of elements of $W$ of the form $r_{\gamma, u}$, where $\gamma$ is an edge-path starting at $s$, forms a Coxeter system for $W_{\Omega}$.

(ii) The map $\pi_{1}\left(\Delta^{\text {odd }}, s\right) \rightarrow W$ given by $\gamma \mapsto p_{\gamma}$ is an isomorphism onto $\Gamma_{\Omega}$.

Remarks. (1) Once we have proven this theorem we will reprove it as the following corollary without assuming the existence of a Vinberg representation. (2) It may happen that $r_{\gamma, u}=r_{\gamma^{\prime}, u^{\prime}}$ even if $(\gamma, u) \neq\left(\gamma^{\prime}, u^{\prime}\right)$. For this reason the Coxeter system consists of the set of elements of $W$ having this form, rather than the set of words themselves. We will work out the equalities of this sort in the next section. (3) This Coxeter system is the one associated to the chamber of $K_{\Omega}$ that contains $s$ 's facet of $K$.

Corollary 6. Suppose $(W, S)$ is any Coxeter system and $s \in S$. Define $W_{\Omega}$ as the subgroup of $C_{W}(s)$ generated by all the reflections it contains except $s$. Define $\Gamma_{\Omega}$ as the subgroup of $W$ generated by the elements $p_{\gamma}$ from (ii) of theorem 5 . Then the conclusions of that theorem hold, and $C_{W}(s)=\langle s\rangle \times\left(W_{\Omega} \rtimes \Gamma_{\Omega}\right)$.

Proof. The corollary is a union of assertions about the subgroups $\left\langle S^{0}\right\rangle$ where $S^{0}$ varies over all finite subsets of $S$ containing $s$. For example, the assertion that $\Sigma:=\left\{r_{\gamma, u}\right\} \subseteq W$ is a Coxeter system for $W_{\Omega}$ says that a certain map to $W_{\Omega}$ from a Coxeter group $W_{\Sigma}$ with generating set $\Sigma$ is an isomorphism. To show surjectivity, consider a reflection in $C_{W}(s)$ other than $s$. It lies in some $\left\langle S^{0}\right\rangle$, hence in the corresponding subgroup $W_{\Omega}^{0}$ of $C_{\left\langle S^{0}\right\rangle}(s)$. Applying theorem 5 shows that it lies in the group generated by $\Sigma \cap\left\langle S^{0}\right\rangle$. Similarly, if $W_{\Sigma} \rightarrow W_{\Omega}$ failed to be injective, then there would be some finite subset $\Sigma^{0} \subseteq \Sigma$ such that $W_{\Sigma^{0}} \rightarrow W_{\Omega}$ failed to be injective. But $\Sigma^{0}$ would lie in $\left\langle S^{0}\right\rangle$ for some finite $S^{0} \subseteq S$, and the failure of injectivity would contradict theorem [5. The rest of our claims follow by similar arguments.

Corollary 7 (Brink 6]). Suppose $(W, S)$ is any Coxeter system and $s \in S$. Then the centralizer $C_{W}(s)$ is the semidirect product of its reflection subgroup by the free group $\pi_{1}\left(\Delta^{\text {odd }}, s\right)$.

Corollary 8. Suppose $(W, S)$ is any Coxeter system, $s \in S$ and $Z$ is a set of edgeloops in $\Delta_{s}^{\text {odd }}$ generating $\pi_{1}\left(\Delta^{\text {odd }}, s\right)$. Suppose given edge-paths $\delta_{t}$ in $\Delta_{s}^{\text {odd }}$ from $s$ to $t$, for each $t \in \Delta_{s}^{\text {odd }}$. Then the $p_{\gamma \in Z}$ generate $\Gamma_{\Omega}$ and together with the $r_{\delta_{t}, u}$ they generate $W_{\Omega} \rtimes \Gamma_{\Omega}$. In particular, if $S$ is finite then $C_{W}(s)$ is finitely generated. 
The rest of this section is devoted to proving theorem 5. The focus in the covering space argument in proposition 3 was on $K_{\Omega}^{\circ}$, but now we focus on $K_{\Omega}$ to avoid fussing over the missing faces. By a tile we mean a facet (of some $W$ chamber) that lies in $K_{\Omega}$. Its type means its image in $K=U / W$, which is a facet of $K$. To avoid confusion we write $F_{t}$ for the facet of $K$ corresponding to $t \in S$. By proposition 3 the tiles correspond to the nodes of the universal cover $\tilde{\Delta}_{s}^{\text {odd }}$, and their types to the nodes of $\Delta_{s}^{\text {odd }}$. By the base tile we mean the tile $K \cap K_{\Omega}$, for which we already have the name $F_{s}$. Now we can explain the words $p_{\gamma}$ :

Lemma 9. Suppose $T$ is a tile, $\left(T_{0}=F_{s}, T_{1}, \ldots, T_{n}=T\right)$ is an edge-path in $\tilde{\Delta}_{s}^{\text {odd }}$ from the base tile to it, and $\gamma=\left(t_{0}=s, \ldots, t_{n}\right)$ is its projection to $\Delta_{s}^{\text {odd }}$. Then $p_{\gamma}$ sends $K$ to the unique chamber in $\frac{1}{2} V$ having $T$ as a facet, and it sends $F_{t_{n}}$ to $T_{n}$.

Proof. The key claim is the following: the image of $F_{t_{n}}$ under $\left(t_{n} t_{n-1}\right)^{l_{n}}$ lies in $t_{n-1}$ 's mirror and its intersection with $F_{t_{n-1}}$ is $F_{t_{n-1}} \cap F_{t_{n}}$; furthermore, $K$ and $\left(t_{n} t_{n-1}\right)^{l_{n}}(K)$ lie on the same side of this mirror. The proof is a picture-drawing exercise in a dihedral group of twice odd order (cf. [1, Fig. 3]).

The lemma is just this claim wrapped inside an induction. The case $n=0$ is trivial, so take $n>0$ and let $\beta$ be the subpath $\left(T_{0}, \ldots, T_{n-1}\right)$ of $\gamma$. The induction hypothesis tells us that $p_{\beta}$ sends $F_{t_{n-1}}$ to $T_{n-1}$ and $K$ into $\frac{1}{2} V$. Conjugating the previous paragraph's claim by $p_{\beta}$ tells us that $p_{\beta} \circ\left(t_{n} t_{n-1}\right)^{l_{n}} \circ p_{\beta}^{-1}$ has two properties. First, it sends $p_{\beta}\left(F_{t_{n}}\right)$ into the mirror containing $p_{\beta}\left(F_{t_{n-1}}\right)=T_{n-1}$ (this mirror is $\left.V^{s}\right)$ and on the other side of $p_{\beta}\left(F_{t_{n-1}} \cap F_{t_{n}}\right)$ from $T_{n-1}$. Second, it sends $p_{\beta}(K)$ into the same side of $V^{s}$ containing $p_{\beta}(K)$. These two statements unravel to give $p_{\gamma}\left(F_{t_{n}}\right)=T_{n}$ and $p_{\gamma}(K) \subseteq \frac{1}{2} V$.

Now we address $W_{\Omega}$. Given a tile $T$ of type $t, T$ 's facets correspond to intersections of $F_{t}$ with other facets $F_{u}$ of $K$. Given a facet $F_{u \neq t}$ of $K$ that meets $F_{t}$, we write $[T, u]$ for the corresponding facet of $T$. If $F_{t}, F_{u}$ make angle $\pi /$ (odd), then by proposition 3 there is another tile on the other side of $[T, u]$ sharing that facet. On the other hand, if the angle is $\pi /$ (even) then we know from the same proposition that $[T, u]$ is not in $K_{\Omega}^{\circ}$, so it lies in a facet of $K_{\Omega}$. That is, $W_{\Omega}$ contains a reflection across $[T, u]$. Every facet of $K_{\Omega}$ contains some such $[T, u]$. The discussion after theorem 11 tells us that the reflections obtained this way form a Coxeter system for $W_{\Omega}$. These reflections are just the $r_{\gamma, u}$ 's:

Lemma 10. Suppose $T$ is a tile of type $t$ and $u \in S$ is evenly joined to $t$. Suppose $\left(T_{0}=F_{s}, T_{1}, \ldots, T_{n}=T\right)$ is a path in $\tilde{\Delta}^{\text {odd }}$, and $\gamma$ the corresponding path in $\Delta^{\text {odd }}$. Then $r_{\gamma, u}$ lies in $W_{\Omega}$ and is the reflection whose mirror contains $[T, u]$.

Proof. This is similar to lemma 9. recall from the definition of $r_{\gamma, u}$ that we write $2 \lambda$ for the edge label between $t_{n}$ and $u$. The key claim is that $\left(u t_{n}\right)^{\lambda-1} u$ is the reflection in $\left\langle t_{n}, u\right\rangle$ that centralizes $t_{n}$ (other than $t_{n}$ itself). This is a slightly different picture-drawing argument than before. The lemma follows from this claim just as before.

Proof of theorem 5. Before lemma 10 we explained how the facets of $K_{\Omega}$ are covered by the $[T, u]$, and this lemma tells us that the $r_{\gamma, u}$ 's are the reflections across them. We know from theorem 1 that these reflections form a Coxeter system for $W_{\Omega}$, proving part $(i)$. 
For part $(i i)$ we note that an element of $\Gamma_{\Omega}$ sends $F_{s}$ to some tile, hence equals $p_{\gamma}$ for some path $\gamma$ in $\Delta^{\text {odd }}$ based at $s$. This equality uses the simple transitivity of $W$ on chambers. If the endpoint of $\gamma$ in $\Delta^{\text {odd }}$ is $t$, then lemma 9 shows that $p_{\gamma}$ conjugates $t$ to $s$. Since membership in $\Gamma_{\Omega}$ requires commutativity with $s$, this shows $t=s$. So $p_{\gamma}$ can only lie in $\Gamma_{\Omega}$ if it is a loop. Conversely, if $\gamma$ is a loop then the lemma shows that $p_{\gamma}$ sends $F_{s}$ into $V^{s}$ and hence centralizes $s$. This shows that the $p_{\gamma}$ 's with $\gamma$ a loop generate $\Gamma_{\Omega}$, and the freeness of $\Gamma_{\Omega}$ comes from theorem 4 .

\section{The Coxeter diagram of $W_{\Omega}$}

We showed in theorem 1 that $W_{\Omega}$ is a Coxeter group, and described a Coxeter system for it in corollary [6. Our goal in this section is to work out the Coxeter diagram $\Delta_{\Omega}$ in a manner making obvious the action of $\Gamma_{\Omega}$. This gives a complete answer to the problem of presenting reflection centralizers in Coxeter groups. We will use the geometric language of Vinberg representations, but the results transfer to general Coxeter groups by the methods used for corollary 6 .

Recall from section 2 that a tile means a facet (of some $W$-chamber) that lies in $K_{\Omega}$. By an arrow we mean a facet of a tile, that is not a facet of any other tile, i.e., it lies in the boundary of $K_{\Omega}$. This peculiar terminology helps organize the calculations in examples; see section 4. The arrows fall into equivalence classes according to which facet of $K_{\Omega}$ they lie in, which we call the arrow classes. To describe $\Delta_{\Omega}$ we must find the arrow classes and understand how the corresponding facets of $K_{\Omega}$ meet.

Whenever we use a symbol with a tilde, such as $\tilde{A}$, for a node of $\tilde{\Delta}_{s}^{\text {odd }}$, we will use the corresponding symbol without the tilde for its image in $\Delta^{\text {odd }}$. We explained in section 2 that the arrows are in bijection with the pairs $[\tilde{A}, B]$, where $\tilde{A}$ and $B$ are vertices of $\tilde{\Delta}_{s}^{\text {odd }}$ and $\Delta$ respectively, and the edge joining $A$ and $B$ in $\Delta$ is evenly-labeled (which includes the case that the edge is absent). The next step is to determine the dihedral angles among the arrows. (If two arrows meet in codimension 1 then we say they make dihedral angle $\pi / n$ as a shorthand for the product of their reflections having order $n$. This order may be 1 because they may lie in the same facet of $K_{\Omega}$.)

Lemma 11. If $\tilde{A}$ is a vertex of $\tilde{\Delta}_{s}^{\text {odd }}$ and $B$ and $C$ are vertices of $\Delta$, such that the subdiagram of $\Delta$ formed by $A, B$ and $C$ appears in table 1 , then the indicated arrows intersect in codimension 1 , with the stated dihedral angle. Conversely, if two arrows meet in codimension 1 then there exist such $\tilde{A}, B, C$, such that the arrows are the ones indicated in the table.

Proof. Suppose $[\tilde{A}, B]$ and $\left[\tilde{A}^{\prime}, B^{\prime}\right]$ are arrows, whose intersection $\phi$ has codimension one in each. Write $K$ for the chamber associated to $\tilde{A}$. Then $\phi$ is a codimension 3 face of $K$, so it corresponds to a spherical 3-vertex subdiagram of $\Delta_{K}$, containing $A$ and $B$. Write $Y$ for the corresponding finite Coxeter group and $C$ for the third vertex. We next verify the conclusions of the theorem if this subdiagram appears in table 1 .

The calculation takes place entirely in the standard representation of $Y$, which we think of as transverse to $\phi$. In this $\mathbb{R}^{3}, U^{s}$ appears as a hyperplane $H, \frac{1}{2} V$ as a half-space bounded by $H, K$ as a chamber of $Y$ with a facet in $H$, and $\tilde{A}$ equal to 


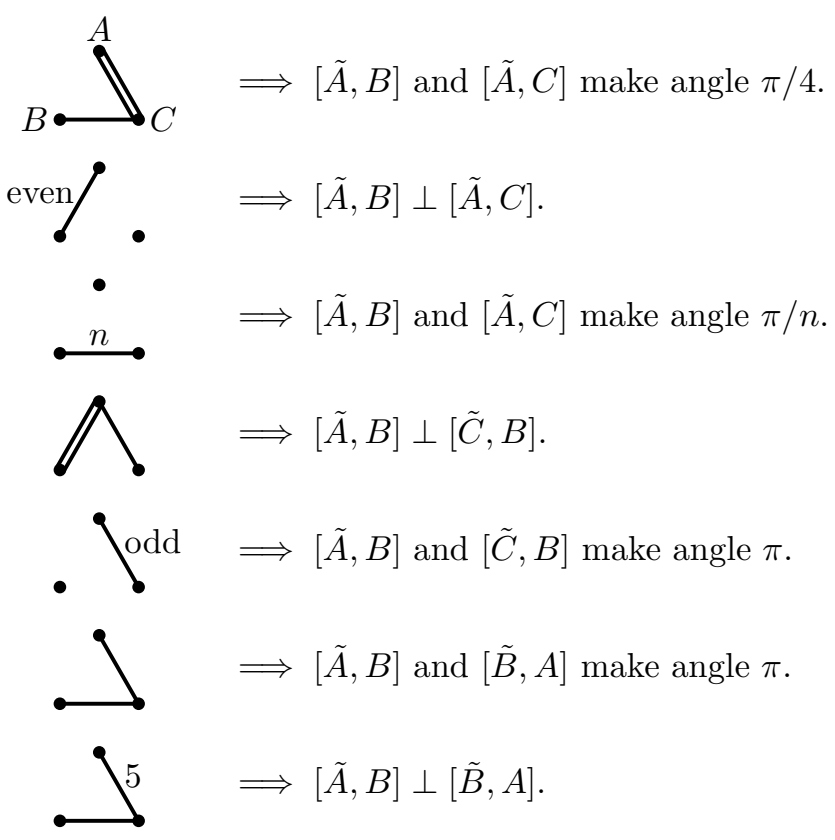

TABLE 1. Dihedral angles between arrows (facets of tiles); see lemma 11] We implicitly label the vertices of all the diagrams $A, B, C$ as in the first one. In the last four lines $\tilde{C}$ means the tile of type $C$ adjacent to $\tilde{A}$, and in the last two lines $\tilde{B}$ means the tile of type $B$ adjacent to $\tilde{C}$.

this facet. The two other facets of this chamber correspond to $B$ and $C$. We write $F_{A}, F_{B}$ and $F_{C}$ for these facets, and $R_{A B}, R_{B C}$ and $R_{C A}$ for the rays where these facets meet.

The simplest case is when $A$ and $C$ are evenly joined. Then $[\tilde{A}, C]$ is also an arrow containing $\phi$, so it is the only one other than $[\tilde{A}, B]$. Also, $[\tilde{A}, B]$ and $[\tilde{A}, C]$ correspond to $R_{A B}$ and $R_{A C}$, so the angle between them is the angle between these rays in $\mathbb{R}^{3}$.

The next case is when $A$ and $C$ are oddly joined and $C$ and $B$ are unjoined or evenly joined. Let $\Theta$ be the rotation around $R_{C A}$ with $\Theta\left(F_{C}\right)$ in $H$ but not overlapping $F_{A}$. Then $\tilde{C}$ corresponds to $\Theta\left(F_{C}\right)$, and $[\tilde{C}, B]$ is the arrow meeting $[\tilde{A}, B]$ in $\phi$; it corresponds to $\Theta\left(R_{B C}\right)$. So the angle between these arrows is the angle in $\mathbb{R}^{3}$ between $R_{A B}$ and $\Theta\left(R_{B C}\right)$. (This rotation process is the reverse of the gift-wrapping process of section 1.)

In the final case, $A$ and $C$ are oddly joined and so are $C$ and $B$. We will apply a second rotation. Namely, let $\Theta^{\prime}$ be the rotation around $\Theta\left(R_{B C}\right)$ with $\Theta^{\prime} \circ \Theta\left(F_{B}\right)$ in $H$ but not overlapping $\Theta\left(F_{C}\right)$. Then $\tilde{B}$ corresponds to $\Theta^{\prime} \circ \Theta\left(F_{B}\right)$, and $[\tilde{B}, A]$ is the arrow meeting $[\tilde{A}, B]$ in $\phi$; it corresponds to $\Theta^{\prime} \circ \Theta\left(R_{A B}\right)$. The angle between these arrows is the angle in $\mathbb{R}^{3}$ between $R_{A B}$ and $\Theta^{\prime} \circ \Theta\left(R_{A B}\right)$.

One can find these angles without computation. Consider the edges $E_{A}, E_{B}$ and $E_{C}$ of the spherical triangle defined by $F_{A}, F_{B}$ and $F_{C}$. In the three cases the desired angle is $\ell\left(E_{A}\right), \ell\left(E_{A}\right)+\ell\left(E_{C}\right)$ and $\ell\left(E_{A}\right)+\ell\left(E_{B}\right)+\ell\left(E_{C}\right)$, where $\ell$ indicates 
length. Drawing the tessellation of the sphere by $Y^{\prime}$ 's chambers makes it easy to recognize which submultiple of $\pi$ this is. This justifies the entries in table 1 .

The table is complete because one can write down all possibilities for the spherical diagram on $A, B$ and $C ; A$ and $B$ should be evenly joined since $[\tilde{A}, B]$ is an arrow. The possibilities other than those in table 1 are

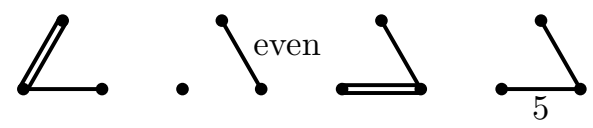

where we use the table's labeling of vertices. The first and second differ from (11) and (2) by $B \leftrightarrow C$, the third from (41) by $A \leftrightarrow C$ and the last from (7) by $A \leftrightarrow B$. In all cases the conclusion of the relevant line of the table is symmetric under the same interchange. So if the diagram of $A, B, C$ doesn't appear in the table then we just swap $[\tilde{A}, B]$ and $\left[\tilde{A}^{\prime}, B^{\prime}\right]$.

The lemma allows one to compute the Coxeter diagram $\Delta_{\Omega}$ :

Theorem 12. The equivalence relation of arrows lying in the same facet of $K_{\Omega}$ is generated by the equivalence of $[\tilde{A}, B]$ with $[\tilde{C}, B]$ in the situation of (5) and that of $[\tilde{A}, B]$ with $[\tilde{B}, A]$ in the situation of ([6).

If two arrow classes have representative arrows as in one of the other entries of the table, then the corresponding facets of $K_{\Omega}$ have the listed dihedral angle. If they have no such representatives then they do not meet, and the edge of $\Delta_{\Omega}$ joining them is labeled $\infty$.

Proof. If two arrows lie in the same facet of $K_{\Omega}$ then there is a chain of arrows joining them, each lying in that facet of $K_{\Omega}$ and meeting the next in codimension 1. This proves the first claim. The second follows immediately from lemma 11, and the third is obvious.

We close the section with a few remarks on the language. We visualize $[\tilde{A}, B]$ as an arrow pointing from the vertex $\tilde{A}$ of $\tilde{\Delta}_{s}^{\text {odd }}$ to the vertex $B$ of $\Delta$. We define a tail class as an equivalence class of facets under the relation (5), i.e., $[\tilde{A}, B]$ and $[\tilde{C}, B]$ are equivalent when $A, B, C$ form the configuration (5). The reason for the name is that the equivalence corresponds the tail moving around in $\tilde{\Delta}_{s}^{\text {odd }}$ while the head stays fixed in $\Delta$.
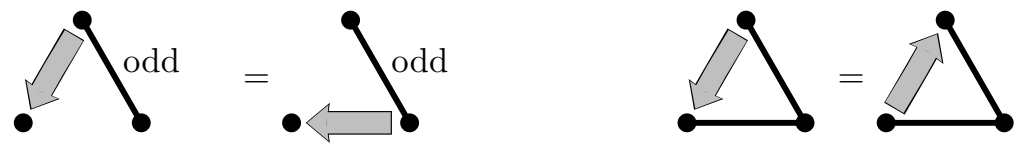

The second picture is a graphical interpretation of (6), although one must be careful keeping track of which vertices lie in $\tilde{\Delta}_{s}^{\text {odd }}$ and which lie in $\Delta$. In most of our examples in the next section $\Delta_{s}^{\text {odd }}$ will be a tree, so $\tilde{\Delta}_{s}^{\text {odd }}$ may be regarded as a subdiagram of $\Delta$. Then we can take the figure literally.

\section{EXAMPLES}

In this section we give many examples of reflection centralizers in Coxeter groups, illustrating theorem 12, See [1] for some different and very nice examples worked from another perspective. We generally proceed by working out the tail classes, fusing them into arrow classes, and then finding the angles. 
Suppose first that $\Delta$ is a tree of single edges (edge label 3). Then there is only one class of reflection, so we don't need to choose a component of $\Delta^{\text {odd }}$. The tail classes are easy to work out: each contains a unique arrow $[A, B]$ where $A$ and $B$ have distance 2 in $\Delta$. (Proof: move the tail toward the head.) The tail classes fuse in pairs, got by reversing these arrows. The end result is that the generators for $W_{\Omega}$ are in bijection with the $A_{3}$ diagrams in $\Delta$. Almost all the angles can be worked out using (3). In our situation it reads: if two arrow classes have representatives with the same tail, then their edge label in $\Delta_{\Omega}$ is the same as the one between their tips in $\Delta$.

The first example is $A_{n \geq 3}$, which has $n-2$ arrow classes:

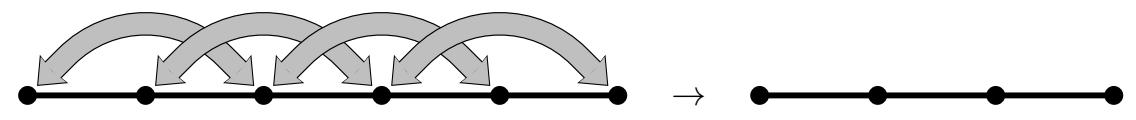

We have drawn double-headed arrows because of the fusion of tail classes. Every arrow class has a representative with tail at the leftmost vertex. So the joins between these arrow classes are the same as the joins between the right-hand tips of the arrows. So $W_{\Omega}=W\left(A_{n-2}\right)$.

The second example is $D_{n \geq 6}$, which has $n-1$ arrow classes:

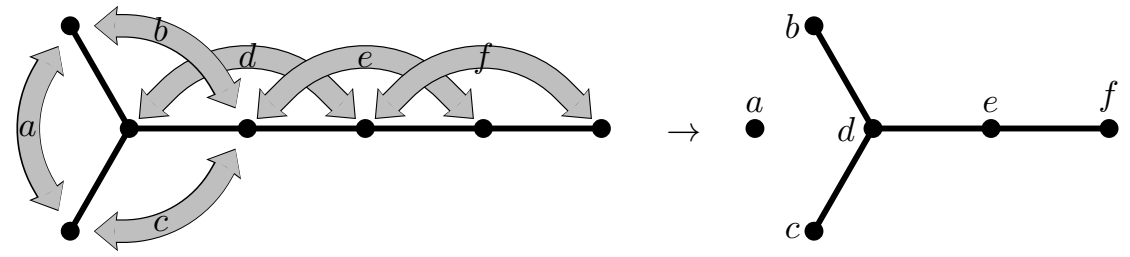

Choosing representative arrows with tails at the top left shows that $a$ is orthogonal to all the other generators except perhaps $c$. Repeating the argument with tails at the lower left shows that $a$ is also orthogonal to $c$. Then taking tails at the rightmost vertex shows that $b, \ldots, f$ form a $D_{n-2}$ diagram. So $W_{\Omega}=W\left(A_{1} D_{n-2}\right)$. The $D_{4}$ and $D_{5}$ cases are the same provided one interprets $D_{2}$ and $D_{3}$ as $A_{1}^{2}$ and $A_{3}$.

The third example is the affine diagram $\tilde{D}_{n \geq 6}$, which gives $W_{\Omega}=W\left(\tilde{A}_{1} \tilde{D}_{n-2}\right)$ in a similar way:

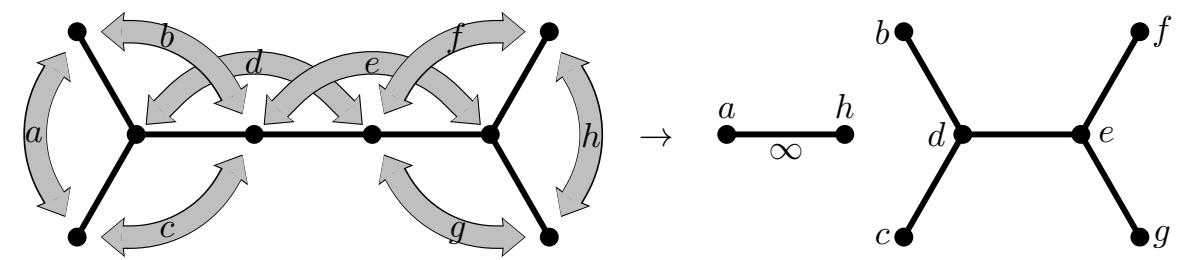

The new phenomenon is that the arrows $a$ and $h$ cannot be moved into a spherical 3 -vertex diagram. That is, their arrow classes contain no representatives lying in such a diagram. (In fact each arrow is its entire class.) So those facets of $K_{\Omega}$ don't meet, hence the edge label $\infty$. The $\tilde{D}_{4}$ and $\tilde{D}_{5}$ cases are the same, provided one interprets $\tilde{D}_{2}$ and $\tilde{D}_{3}$ as $\tilde{A}_{1}^{2}$ and $\tilde{A}_{3}$.

These examples are enough to treat the general case:

Theorem 13. If $\Delta$ is a tree of single edges, then the vertices of $\Delta_{\Omega}$ are the $A_{3}$ subdiagrams of $\Delta$, with edge labels as follows. If the convex hull of two $A_{3}$ 's in $\Delta$ 
has type $D$ (resp. $\tilde{D})$, then their edge label in $\Delta_{\Omega}$ is 2 (resp. $\left.\infty\right)$. Otherwise, it is the same as the one in $\Delta$ between their middle vertices.

In the special case of trivalent branch points, no two adjacent, $\Delta_{\Omega}$ can be got from $\Delta$ by the following operation: "blow up" each branch point

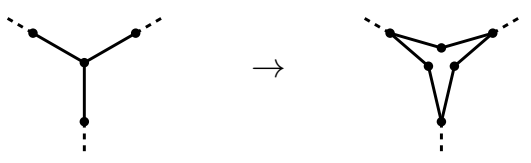

and then erase all the end vertices of $\Delta$, and finally add some edges labeled $\infty$. When there is only one branch point there are no $\infty$ 's. For example, the $Y_{555}$ diagram gives

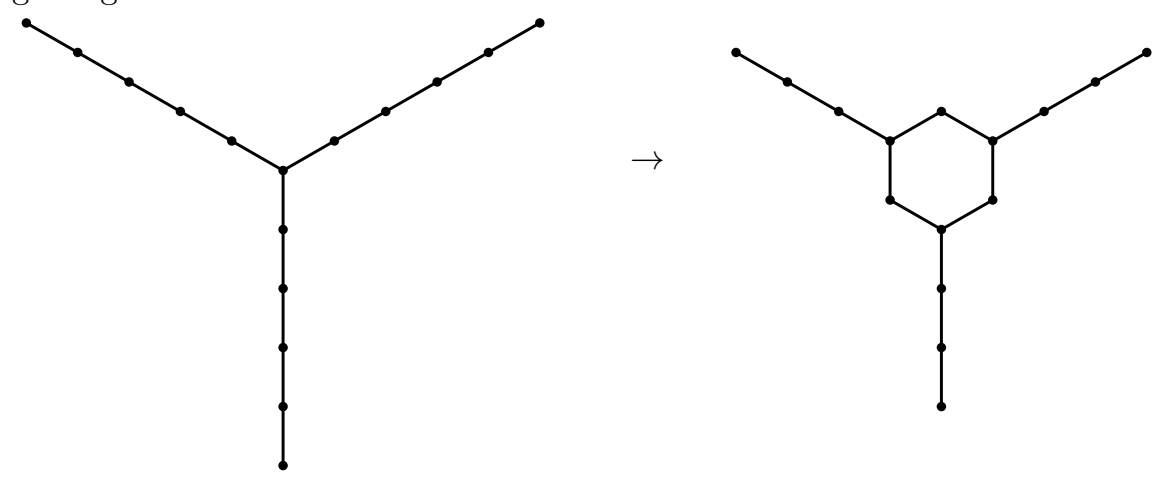

We chose this example because it explains the appearance of the latter figure in the ATLAS [10] entry for the monster simple group $M$, given the appearance of the former. Namely, the bimonster $(M \times M): 2$ is described as a quotient of the $Y_{555}$ Coxeter group, and $M \times 2$ as a quotient of the Coxeter group of the second figure. Given the first, one should expect the second, because a $Y_{555}$ reflection maps to an involution in the bimonster with centralizer $M \times 2$. (One can repeat the process, so the reflection centralizer in the second diagram maps to the involution centralizer $2 B \times 2 \subseteq M \times 2$ where $B$ is the baby monster. Since the nonreflection part is now $\mathbb{Z}$, the $Y_{555}$ approach to $M$ distinguishes a conjugacy class in $B$, up to inversion. I don't know what class this is or whether this approach offers any real insight.)

Another example is $\Delta=E_{8}$, which we show in several steps to illustrate the interaction between blowing up branch points and erasing ends:

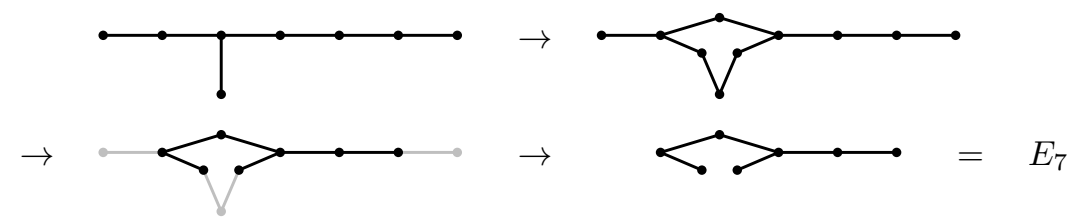

The first step blows up the branch point, the second shows what will be erased, and the third actually erases it.

An example with an edge label $\infty$ is the reflection group of the even unimodular lattice of signature $(17,1)$. By [14] (see also [9]), $\Delta$ is

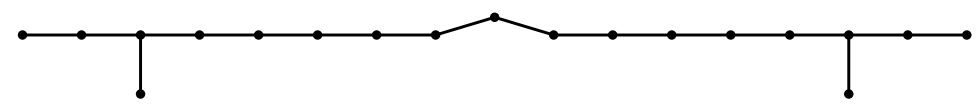


which after explosion and erasure yields

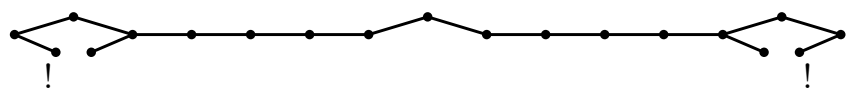

Since the vertices marked "!" correspond to $A_{3}$ 's in $\Delta$ whose convex hull is a $\tilde{D}_{16}$, they should be joined by an edge labeled $\infty$. Adjoining this edge completes the description of $\Delta_{\Omega}$. Remarks: (1) this is the reflection group of the even lattice of signature $(16,1)$ and determinant -2. (2) Because it acts on hyperbolic space $H^{16}$, it makes sense to ask whether this $\infty$ represents parallelism or ultraparallelism. It represents parallelism, because the corresponding infinite dihedral group lies in the affine group $W\left(\tilde{D}_{16}\right)$.

As a meatier example we treat a Coxeter group found by Bugaenko [8]. It acts cocompactly on $H^{8}$, and is the only known cocompact example on any $H^{n \geq 8}$. Here $\Delta$ is

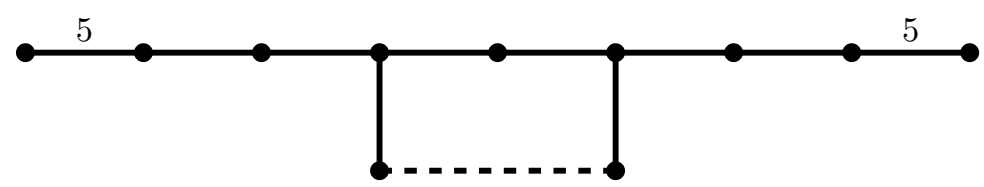

where the dashed line means an edge label $\infty$. The same argument as before shows that every tail class is represented by a unique arrow from one vertex to another at distance 2 . So we can name the 13 generators of $W_{\Omega}$ :

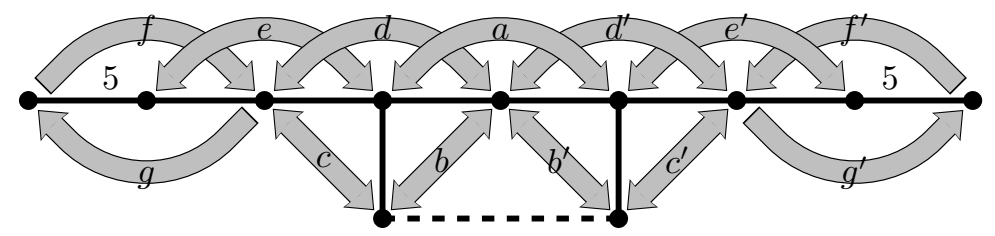

Note that $f$ and $g$ represent distinct arrow classes, because only " $A_{3}$ arrows" are reversible. Taking tails at the left end of $\Delta$ shows that $f, e, d, c, a, b^{\prime}, d^{\prime}, e^{\prime}, g^{\prime}$ are joined the same way as their right endpoints are joined in $\Delta$. Exchanging primed and unprimed letters gives all joins among $f^{\prime}, e^{\prime}, d^{\prime}, c^{\prime}, a, b, d, e, g$, so we know all the joins except those between a member of $\left\{g, b, c^{\prime}, f^{\prime}\right\}$ and a member of $\left\{g^{\prime}, b^{\prime}, c, f\right\}$. By the priming symmetry there are only 10 cases left to work out. Taking tails based at the middle vertex gives $b b^{\prime}=\infty$ and $g g^{\prime}=2$. Taking tails based at the lower left vertex gives $b c=b g^{\prime}=c g=2$. We have $f g=2$ by (7). Finally, we have $b f=c c^{\prime}=c f^{\prime}=f f^{\prime}=\infty$ because in none of these cases is there a 3-vertex spherical diagram containing representatives for both arrow classes. So $\Delta_{\Omega}$ is

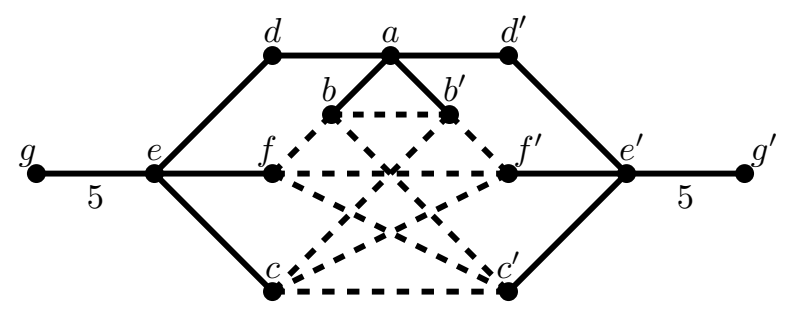

We are grateful to one of the referees for suggesting the following unpublished example of Howlett: the reflection centralizer when $\Delta$ is a 4-cycle of edges labeled 5 . It turns out that $W_{\Omega}$ has countably many generators but no relations at all. One 
can see this as follows. Here $\Delta^{\text {odd }}$ is a cycle, so $\tilde{\Delta}^{\text {odd }}$ is an infinite chain. An arrow has its tail at a point of this chain and its tip in $\Delta$, evenly joined to the projection of the tail. There is only candidate for the tip, so there is one arrow for each point of the chain. None of the diagrams from table 1 appear in $\Delta$, so there is no fusion into arrow classes and there are no relations between the arrows.

An interesting twist on this example is when $\Delta$ is a pentagon with edges labeled 5 . For each pair of adjacent points of the chain $\tilde{\Delta}^{\text {odd }}$ there is a unique point of $\Delta$ to which they both have arrows. These pairs are the tail classes, which are also the arrow classes because there are no $A_{3}$ diagrams. Given three consecutive points of $\tilde{\Delta}^{\text {odd }}$, the first two give an arrow class and so do the last two. These arrow classes have representatives with the same tail (the middle point), so they are joined in $\Delta_{\Omega}$ the same way their tips are joined in $\Delta$, namely by an edge labeled 5 . The end result is that $\Delta_{\Omega}$ is an infinite chain with adjacent (resp. nonadjacent) nodes joined by edges labeled 5 (resp. $\infty$ ). Pursuing this to its logical conclusion yields the following:

Theorem 14. Suppose $\Delta$ is a cycle of $n>3$ odd edges, with nodes labeled $s_{0}, \ldots$, $s_{n-1}$ in cyclic order and subscripts read modulo $n$. Then $W_{\Omega}$ has generators $S_{i \in \mathbb{Z}}$ and relations

(i) $\left(S_{i} S_{j}\right)^{o\left(s_{i} s_{j}\right)}=1$ if $|i-j| \leq n-4$

(ii) $\left(S_{i} S_{i+2-n}\right)^{2}=1$ if $\left\{s_{i}, s_{i+1}, s_{i+2}\right\}$ has type $H_{3}$

(iii) $S_{i}=S_{i+2-n}$ if $\left\{s_{i}, s_{i+1}, s_{i+2}\right\}$ has type $A_{3}$

A generator for $\Gamma_{\Omega}$ acts by $S_{i} \mapsto S_{i+n}$.

Remarks. (1) If $n \leq 3$ then $W_{\Omega}$ is trivial. (2) This is not quite a Coxeter presentation since the third set of relations may identify generators with each other. After this identification one does obtain a Coxeter presentation. (3) The affine diagram $\tilde{A}_{n-1}$ is a cycle of $n$ edges labeled 3 . In this case the third set of relations read $S_{i}=S_{i+2-n}$ for all $i$, so the centralizer has $n-2$ generators. The first set of relations shows that $W_{\Omega}$ is the affine group $\tilde{A}_{n-3}$. A generator for $\Gamma_{\Omega}$ acts on this $\tilde{A}_{n-3}$ diagram by rotation by two notches.

Proof sketch. Call $\tilde{\Delta}^{\text {odd }}$ 's nodes $t_{i \in \mathbb{Z}}$, where $t_{i}$ projects to $s_{i}$. An arrow has the form $\left[t_{i}, s_{j}\right]$ where $i \not \equiv j, j \pm 1 \bmod n$. The tail classes are

$$
S_{i}=\left\{\left[t_{i+2}, s_{i}\right],\left[t_{i+3}, s_{i}\right], \ldots,\left[t_{i+n-2}, s_{i}\right]\right\}
$$

for $i \in \mathbb{Z}$. If $\left|i-i^{\prime}\right| \leq n-4$ then $S_{i}$ and $S_{i^{\prime}}$ contain arrows with the same tail, hence the relation $\left(S_{i} S_{i^{\prime}}\right)^{o\left(s_{i} s_{i^{\prime}}\right)}=1$.

If $\left\{s_{j}, s_{j+1}, s_{j+2}\right\}$ has type $H_{3}$, then (7) says that $\left[t_{i+2}, s_{i}\right]$ and $\left[t_{i}, s_{i+2}\right]$ commute, whenever $i \in \mathbb{Z}$ reduces to $j$ modulo $n$. Since $\left[t_{i+2}, s_{i}\right] \in S_{i}$ and $\left[t_{i}, s_{i+2}\right] \in S_{i+2-n}$, this gives $\left(S_{i} S_{i+2-n}\right)^{2}=1$. If $\left\{s_{j}, s_{j+1}, s_{j+2}\right\}$ has type $A_{3}$ then the same argument using (6) gives $S_{i}=S_{i+2-n}$ instead. The cases (11), (2) and (4) from table 11 are irrelevant.

As a final example we mention Vinberg's diagrams [14, p. 34] for the reflection groups of the odd unimodular Lorentzian lattices. If $s$ is a node of one of these diagrams corresponding to a norm 1 root then $W_{\Omega}$ is the reflection group of its orthogonal complement, which is unimodular Lorentzian of one dimension less. One possibility for $s$ (usually the only one) leads to $W_{\Omega}$ being the previous entry in Vinberg's table. By taking centralizers of nodes corresponding to norm 2 roots one 
could instead rederive the diagrams for the reflection groups of the odd bimodular Lorentzian lattices [14, p. 32].

\section{REFERENCES}

[1] Alekseevski, D., Michor, P. and Neretin, Y., Rolling of Coxeter polyhedra along mirrors, to appear in Geometric Methods in Physics: XXXI Workshop, eds. Kielanowski et. al., Birkaüser.

[2] Allcock, Daniel, Normalizers of parabolic subgroups of Coxeter groups, Algebraic 6 Geometric Topology 12 (2012) 11371143.

[3] Allcock, Daniel, Steinberg groups as amalgams, in preparation.

[4] Borcherds, Richard E., Coxeter groups, Lorentzian lattices, and K3 surfaces, Internat. Math. Res. Notices 1998, no. 19, 1011-1031.

[5] Bourbaki, Nicolas, Éléments de mathématique. Groupes et algèbres de Lie. Chapitres 4, 5 et 6. Masson, Paris, 1981. English translation: Lie groups and Lie algebras. Chapters 46, Springer-Verlag, Berlin, 2002

[6] Brink, Brigitte, On centralizers of reflections in Coxeter groups, Bull. London Math. Soc. 28 (1996) no. 5, 465-470

[7] Brink, Brigitte and Howlett, Robert B., Normalizers of parabolic subgroups in Coxeter groups, Invent. Math. 136 (1999) no. 2, 323-351.

[8] Bugaenko, V. O., Arithmetic crystallographic groups generated by reflections, and reflective hyperbolic lattices, Lie Groups, Their Discrete Subgroups, and Invariant Theory, Adv. Sov. Math. 8, (1992) 33-55, American Math. Soc., Providence, RI

[9] Conway, cf [11] Conway, J. H, The automorphism group of the 26-dimensional even unimodular Lorentzian lattice, J. Algebra 80 (1983), no. 1, 159-163. Reprinted as ch. 27 of 11

[10] Conway, J. H.; Curtis, R. T.; Norton, S. P.; Parker, R. A.; Wilson, R. A., Atlas of finite groups. Maximal subgroups and ordinary characters for simple groups. With computational assistance from J. G. Thackray. Oxford University Press, Eynsham, 1985.

[11] Conway, J. H., Sloane, N. J. A., et. al., Sphere Packings, Lattices and Groups, SpringerVerlag, 1993.

[12] Howlett, Robert B., Normalizers of parabolic subgroups of reflection groups, J. London Math. Soc. (2) 21 (1980), no. 1, 62-80.

[13] Vinberg, È. B., Discrete linear groups generated by reflections, Math. U.S.S.R. Izvestija 5 (1971) 1083-1119.

[14] Vinberg, È. B., The groups of units of certain quadratic forms, Math. U.S.S.R. Sbornik 16 (1972) 17-35.

Department of Mathematics, University of Texas at Austin, Austin, TX 78713

E-mail address: allcock@math.utexas.edu

$U R L:$ http://www. math. utexas. edu/ ${ }^{\sim}$ allcock 